Int. J. Electrochem. Sci., 12 (2017) $8021-8032$

\title{
Sensitive and Low-potential Electrochemical Detection of Hydroquinone Using a Nanodiamond Modified Glassy Carbon Electrode
}

Tse-Wei Chen ${ }^{1}$, Selvakumar Palanisamy ${ }^{1,2}$, Shen-Ming Chen ${ }^{1 *}$, Vijayalakshmi Velusamy ${ }^{2}$, Yung-Hsien Liu $^{3}$, Tien-Wen Tseng ${ }^{1}$, Ming-Chin Yu ${ }^{4}$, Shih-Yi Lee ${ }^{5}$, Wen-Han Chang ${ }^{6,7,8}$, Xiaoheng Liu, ${ }^{9, *}$

${ }^{1}$ Department of Chemical Engineering and Biotechnology, National Taipei University of Technology, Taipei 106, Taiwan (ROC)

${ }^{2}$ Division of Electrical and Electronic Engineering, School of Engineering, Manchester Metropolitan University, Manchester - M1 5GD, United Kingdom.

${ }^{3}$ National Chung-Shan Institute of Science \& Technology, Taiwan (ROC).

${ }^{4}$ Department of Surgery, Chang Gung Memorial Hospital Linkou Branch, Chang Gung University, Taoyuan, Taiwan. (Department of Surgery, Chang Gung Memorial Hospital at Linkou,) No. 5, FuHsing Street, Kweishan, Taoyuan, 333, Taiwan (ROC).

${ }^{5}$ Division of Pulmonary and Critical Care Medicine, Mackay Memorial Hospital; Mackay Junior College of Medicine, Nursing, and Management, Taiwan (ROC).

${ }^{6}$ Department of Emergency Medicine, Mackay Memorial Hospital; Institute of Mechatronic Engineering, National Taipei University of Technology, Taiwan (ROC).

${ }^{7}$ MacKay Memorial College Department of Cardiology, MacKay Memorial Hospital, Taiwan (ROC).

${ }^{8}$ Graduate Institute of Injury Prevention and Control, Taipei Medical University; School of Medicine, Taipei Medical University, Taiwan (ROC).

${ }^{9}$ Key Laboratory of Education Ministry for Soft Chemistry and Functional Materials, Nanjing University of Science and Technology, Nanjing 210094, China.

*E-mail: $\underline{\text { smchen78@ms15.hinet.net; ; xhliu@mail.njust.edu.cn }}$

doi: $10.20964 / 2017.09 .05$

Received: 31 May 2017 / Accepted: 1 July 2017 / Published: 13 August 2017

The present work describes the sensitive and low-potential detection of hydroquinone (HQ) using a glassy carbon electrode (GCE) modified with nanodiamond (ND). The presence of ND was confirmed by high-resolution scanning electron microscopy, FTIR and Raman spectroscopy. The cyclic voltammetry results reveal that ND modified GCE has high electrocatalytic activity towards oxidation of HQ than unmodified GCE. In addition, the ND modified GCE shows a significantly lower oxidation potential towards $\mathrm{CC}$ than unmodified $\mathrm{GCE}$, which is due to the presence of $-\mathrm{OH}$ functional groups in ND. The CV studies confirm that the redox electrochemical behavior of $\mathrm{CC}$ is a diffusion controlled electrochemical process on ND modified electrode. The ND modified electrode was able to detect the 
HQ across a linear response range from 1.0 to $78.0 \mu \mathrm{M}$. The limit of detection of the sensor was 0.19 $\mu \mathrm{M}$. The sensor shows an appropriate selectivity in the presence of 20 -fold concentrations of benzenediols and neurotransmitters. The practicality of the sensor is appropriate and shows excellent recovery of HQ in lab water samples.

Keywords: Nanodiamond; Modified electrode; Cyclic voltammetry; Hydroquinone, Electroanalysis

\section{FULL TEXT}

(C) 2017 The Authors. Published by ESG (www.electrochemsci.org). This article is an open access article distributed under the terms and conditions of the Creative Commons Attribution license (http://creativecommons.org/licenses/by/4.0/). 